\title{
A Receding Horizon Generalization of Pointwise Min-Norm Controllers
}

\author{
James A. Primbs, Vesna Nevistić, and John C. Doyle, Member, IEEE
}

\begin{abstract}
Control Lyapunov functions (CLF's) are used in conjunction with receding horizon control (RHC) to develop a new class of receding horizon control schemes. In the process, strong connections between the seemingly disparate approaches are revealed, leading to a unified picture that ties together the notions of pointwise min-norm, receding horizon, and optimal control. This framework is used to develop a CLF based receding horizon scheme, of which a special case provides an appropriate extension of Sontag's formula. The scheme is first presented as an idealized continuous-time receding horizon control law. The issue of implementation under discrete-time sampling is then discussed as a modification. These schemes are shown to possess a number of desirable theoretical and implementation properties. An example is provided, demonstrating their application to a nonlinear control problem. Finally, stronger connections to both optimal and pointwise min-norm control are proved in the Appendix under more restrictive technical conditions.
\end{abstract}

Index Terms-Control Lyapunov functions, nonlinear optimal control, predictive control, receding horizon control.

\section{INTRODUCTION}

$\mathbf{T}$ HE OPTIMAL control of nonlinear systems is one of the most challenging and difficult subjects in control theory. It is well known that the nonlinear optimal control problem can be reduced to the Hamilton-Jacobi-Bellman (HJB) partial differential equation [2], but due to difficulties in its solution, this is not a practical approach. Instead, the search for nonlinear control schemes has generally been approached on less ambitious grounds than requiring the exact solution to the HJB partial differential equation.

In fact, even the problem of stabilizing a nonlinear system remains a challenging task. Lyapunov theory, the most successful and widely used tool for stability analysis, is a century old. Despite this, systematic methods for obtaining Lyapunov functions for general nonlinear systems still do not exist. Nevertheless, the ideas put forth by Lyapunov nearly a century ago continue to be used and exploited extensively in the modern theory of control for nonlinear systems. One notably successful use of the Lyapunov methodology is its generalization to control systems, known as a control Lyapunov function (CLF) [30], [31], [6], [10], [15], [8], [7]. The knowledge of such a function is suffi-

Manuscript received April 4, 1999; revised July 23, 1999. Recommeded by Associate Editor, M. Krstic.

J. A. Primbs and J. C. Doyle are with the Control and Dynamical Systems 107-81, California Institute of Technology, Pasadena, CA 91125 USA (e-mail: \{jprimbs; doyle\}@cds.caltech.edu).

V. Nevistić is with the Automatic Control Laboratory, ETH, Zürich, Switzerland (e-mail: vesna@aut.ee.ethz.ch).

Publisher Item Identifier S 0018-9286(00)04164-7. cient to design stabilizing control schemes. Once again, systematic techniques for finding CLF's for general nonlinear systems do not exist, but this approach has been applied successfully to many classes of systems for which CLF's can be found [15], [8], [29].

In contrast to the emphasis on guaranteed stability that is the primary goal of CLF's, another class of nonlinear control schemes that go by the names receding horizon, moving horizon, or model predictive control place importance on optimal performance [17], [16], [20], [11], [13]. These techniques apply a receding horizon implementation in an attempt to approximately solve the optimal control problem through on-line computation. For systems under which on-line computation is feasible, receding horizon control (RHC) has proven quite successful [27], [26]. But both stability concerns and practical implementation issues remain a major research focus [20], [23], [3], [24].

Based on their underlying connection with the optimal control problem, in this paper we show that both CLF-based methods and receding horizon control can be cast in a single unifying framework where the advantages of both can be exploited. The strong stability properties of CLF's can be carried into a receding horizon scheme without sacrificing the excellent performance advantages of receding horizon control. With this flexible new approach, computation can be used to its fullest to approach optimality while stability is guaranteed by the presence of the CLF. This approach, in essence, combines and unites the best properties of CLF's and receding horizon control.

The paper is organized as follows. Section II briefly reviews the optimal control problem, CLF-based pointwise min-norm controllers and our variation of Sontag's formula, and the receding horizon methodology. Section III then connects these approaches by providing a unified framework in which to view them. From this common vantage point, a new RHC + CLF scheme is introduced in Section IV. We first present this new scheme in an idealized continuous-time receding horizon framework. Issues of implementation under discrete time sampling are then mentioned as a modification to the presented scheme. Various theoretical and implementation properties possessed by the scheme are discussed, including a special choice of parameters that corresponds to Sontag's formula. This approach is tested on an example in Section V and conclusions are drawn in Section VI. Finally, in the Appendix, technical assumptions including global Lipschitzness are used to prove results demonstrating stronger connections with optimal and pointwise min-norm controllers. 


\section{BACKGROUND}

Let $\mathbb{R}$ denote the reals and $\mathbb{R}_{+}$the nonnegative real numbers. Gradients will be written in shorthand as $V_{x}$, i.e.,

$$
V_{x}=\frac{\partial V}{\partial x}=\left[\frac{\partial V}{\partial x_{1}}, \cdots, \frac{\partial V}{\partial x_{n}}\right] .
$$

We consider nonlinear systems of the form

$$
\dot{x}=f(x)+g(x) u \quad f(0)=0
$$

with $x \in \mathbb{R}^{n}$ denoting the state, $u \in \mathbb{R}^{m}$ the control, and $f(x): \mathbb{R}^{n} \rightarrow \mathbb{R}^{n}$ and $g(x): \mathbb{R}^{n} \rightarrow \mathbb{R}^{n \times m}$ continuously differentiable in all arguments.

\section{A. Nonlinear Optimal Control}

The standard nonlinear optimal control problem is formulated as follows:

\section{(Optimal Control Problem)}

$$
\begin{aligned}
& \inf _{u(\cdot)} \quad \int_{0}^{\infty}\left(q(x)+u^{T} u\right) d \tau \\
& \text { s.t. } \quad \dot{x}=f(x)+g(x) u
\end{aligned}
$$

for $q(x)$ continuously differentiable and positive semidefinite with the desired solution being a state-feedback control law. We will also assume that the system $[f(x), q(x)]$ is zero-state detectable. (i.e., for all $x \in \mathbb{R}^{n}, q(\phi(t, x))=0 \Rightarrow \phi(t, x) \rightarrow 0$ as $t \rightarrow \infty$ where $\phi(t, x)$ is the state transition function of the system $\dot{x}=f(x)$ from the initial condition $x(0)=x$.)

1) Hamilton-Jacobi-Bellman Equations: A standard dynamic programming argument reduces the above optimal control problem to the HJB partial differential equation [2]

$$
V_{x}^{*} f-\frac{1}{4}\left(V_{x}^{*} g g^{T} V_{x}^{* T}\right)+q(x)=0
$$

where $V^{*}$ is commonly referred to as the value function and can be thought of as the minimum cost to go, i.e.,

$$
V^{*}(x(t))=\inf _{u(\cdot)} \int_{t}^{\infty}\left(q(x(\tau))+u^{T}(\tau) u(\tau)\right) d \tau .
$$

If a positive semidefinite, continuously differentiable solution to the HJB equation (3) exists, then the optimal state-feedback control law is given by

$$
u^{*}=-\frac{1}{2} g^{T} V_{x}^{* T} .
$$

Unfortunately, the HJB equation (3) is extremely difficult to solve and, in general, precludes any hope of an exact solution to the optimal control problem.

2) Euler-Lagrange Equations: A related optimal control problem is the finite horizon problem with a terminal weight and specified initial condition:

$$
\begin{array}{ll}
\inf _{u(\cdot)} & \int_{0}^{T}\left(q(x)+u^{T} u\right) d \tau+\varphi(x(T)) \\
\text { s.t. } & \dot{x}=f(x)+g(x) u \\
& x(0)=x_{0} .
\end{array}
$$

This problem is often reduced to the Euler-Lagrange ordinary differential equations [2]

$$
\begin{array}{rlrl}
\dot{x} & =H_{\lambda}\left(x, u^{*}, \lambda\right) & & x(0)=x_{0} \\
\dot{\lambda} & =-H_{x}\left(x, u^{*}, \lambda\right) & \lambda(T)=\varphi_{x}^{T}(x(T)) \\
u^{*} & =\arg \min _{u} H(x, u, \lambda) &
\end{array}
$$

where $H(x, u, \lambda)=q(x)+u^{T} u+\lambda^{T}(f(x)+g(x) u)$ is referred to as the Hamiltonian. These equations represent a necessary condition for optimality and are much easier to solve than the HJB equation. But, this problem is not equivalent to the infinite horizon problem given in (2) unless the terminal weight $\varphi(\cdot)$ is the value function $V^{*}(\cdot)$ (which is found by solving the HJB equation). Furthermore, this problem is solved for a single initial condition and produces an open-loop control law, in contrast to the global, closed-loop solution that the HJB approach provides. For a detailed discussion of both HJB and Euler-Lagrange equations, the reader is referred to [2].

Below, we present two suboptimal approaches to the optimal control problem; the first of which corresponds well to the HJB approach, while the second exploits Euler-Lagrange type trajectory optimizations.

\section{B. Control Lyapunov Functions (CLF's)}

A control Lyapunov function (CLF) is a continuously differentiable, proper, positive definite function $V: \mathbb{R}^{n} \rightarrow \mathbb{R}_{+}$such that

$$
\inf _{u}\left[V_{x}(x) f(x)+V_{x}(x) g(x) u\right]<0
$$

for all $x \neq 0$ [1], [30], [31]. Techniques for the derivation of a CLF is an important research topic and is covered in many references (see, e.g., [29] and references therein). Given a CLF, a stabilizing controller may be designed by posing the following optimization [6], [7]:

\section{(Pointwise Min-Norm)}

$$
\begin{array}{cc} 
& \inf _{u} \quad u^{T} u \\
\text { s.t. } & V_{x}(f(x)+g(x) u) \leq-\sigma(x)
\end{array}
$$

where $\sigma(x)$ is some continuous, positive definite function satisfying $V_{x} f(x) \leq-\sigma(x)$ whenever $V_{x} g(x)=0$. The pointwise (i.e., for each $x$ ) solution to the problem produces a state-feedback control law, referred to as a pointwise min-norm controller [7]. The name derives from the fact that this formula pointwise minimizes the control energy used while requiring that $V$ be a Lyapunov function for the closed-loop system and decrease at a rate of $\sigma$ at every point. Under proper technical conditions, pointwise min-norm controllers are inverse optimal, which means that they correspond to the solution of the HJB equation for a meaningful cost [7]. A particularly special case of pointwise min-norm controllers results when $\sigma(x)$ is chosen as:

$$
\sigma_{s}(x)=\sqrt{\left(V_{x} f\right)^{2}+q(x)\left(V_{x} g g^{T} V_{x}^{T}\right)} .
$$

The result is a slight variation of Sontag's CLF formula, shown in (10)at the bottom of the next page [31].In addition to possessing the continuity properties enjoyed by Sontag's formula 
(i.e., for $q(x)$ positive definite it is continuous everywhere except possibly at $x=0,[31]$ ), it also leads to an interpretation in terms of the optimal control problem. It can be thought of as using the gradient direction of the CLF, but scaled to satisfy the HJB equation. In particular, if the CLF has the same shape level curves as the value function, this formula recovers the optimal controller [10].

\section{Receding Horizon Control (RHC)}

In receding horizon control (cf. [17], [16], [11], [20], and [13]), the current control at state $x$ and time $t$ is obtained by determining on-line the optimal open-loop control trajectory $\hat{u}$ over the interval $[t, t+T]$ respecting the following objective:

\section{(Receding Horizon Control)}

$$
\begin{aligned}
& \inf _{u(\cdot)} \int_{t}^{t+T}\left(q(x(\tau))+u^{T}(\tau) u(\tau)\right) d \tau+\varphi(x(t+T)) \\
& \text { s.t. } \quad \dot{x}=f(x)+g(x) u .
\end{aligned}
$$

The above problem is solved in an open-loop/Euler-Lagrange fashion, exploiting the fact that a solution is needed only for the current state as the initial condition. The optimizing control trajectory $\hat{u}$ is implemented until a new state update is received. These computations are repeated at each time step, updating the initial condition with the current state and resolving and implementing the solution to the above optimization, producing a state-feedback control law.

In essence, receding horizon control attempts to compute the value function and optimal control actions on-line for those states encountered along its trajectory. In this way, receding horizon techniques approach optimal control in a local fashion, as opposed to the more global-in-nature CLF methodologies.

The philosophical underpinnings of CLF and receding horizon techniques lie in the two approaches (HJB and Euler-Lagrange) to the optimal control problem. CLF's can be interpreted as a global approximation to the value function, especially when used in conjunction with Sontag's formula (10) and pointwise min-norm schemes (7), (8). On the other hand, receding horizon control solves Euler-Lagrange type trajectory optimizations and exploits the receding horizon methodology to convert open-loop trajectories into a state-feedback control law.

\section{LIMITS OF RECEDING HORIZON CONTROL}

While CLF's and receding horizon control have connections to the optimal control problem, a deeper look at the actual form of the underlying optimization involved in the following three schemes (optimal control, pointwise min-norm, and receding horizon) leads to even more striking connections. In this section, we develop a heuristic framework for viewing both optimal control (2) and pointwise min-norm control (7), (8) as limiting cases of receding horizon control.

Our starting point will be to consider the standard open-loop optimization that is solved on-line at every time instance in receding horizon control, but without the terminal weight $\varphi(\cdot)$

$$
\int_{t}^{t+T}\left(q(x)+u^{T} u\right) d \tau .
$$

First, we make the trivial observation that as the horizon $T$ tends to infinity, the objective in the optimal control problem (2) is recovered

$$
\int_{t}^{\infty}\left(q(x)+u^{T} u\right) d \tau .
$$

At the other extreme, consider what happens as the horizon $T$ tends to zero. First, note that for any $T$ an equivalent objective function is given by

$$
\frac{1}{T} \int_{t}^{t+T}\left(q(x)+u^{T} u\right) d \tau
$$

since dividing by $T$ has no effect on the optimizing $u$. Now, letting $T \rightarrow 0$ yields

$$
q(x(t))+u^{T}(t) u(t) .
$$

Since $x(t)$ is known, there is no need to include the term $q(x(t))$, leaving

$$
u^{T}(t) u(t)
$$

which is recognized as the objective function used in the pointwise min-norm formulation (7). Hence, this indicates that we may heuristically view the pointwise min-norm problem as a receding horizon optimization with a horizon length of zero. These considerations suggest the following interpretation: optimal control and pointwise min-norm formulations should represent extreme cases of a properly conceived receding horizon scheme. This is pictured in Fig. 1. Ideally, we would hope to incorporate the best properties of each approach into a single scheme parameterized by horizon length. These properties should include:

1) Stability for any horizon $T$.

2) Pointwise min-norm controllers for $T=0$.

3) Optimality for $T=\infty$.

Additionally, there should exist an extension of Sontag's formula that will recover the optimal controller if the level curves of the CLF correspond to those of the value function, regardless of the horizon length $T$. With these goals in mind, we present a new class of CLF-based receding horizon control schemes.

$$
u_{\sigma_{s}}= \begin{cases}-\left[\frac{V_{x} f+\sqrt{\left(V_{x} f\right)^{2}+q(x)\left(V_{x} g g^{T} V_{x}^{T}\right)}}{V_{x} g g^{T} V_{x}^{T}}\right] g^{T} V_{x}^{T}, & V_{x} g \neq 0 \\ 0, & V_{x} g=0\end{cases}
$$




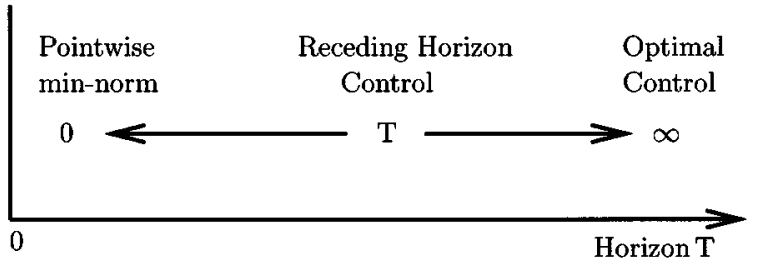

Fig. 1. Unified framework.

\section{ReCEding Horizon Generalization of Pointwise MIN-NORM CONTROLLERS}

In this section, a new class of control schemes is introduced that retain the global stability properties of CLF methods while taking advantage of the on-line optimization techniques employed in receding horizon control. In essence, it represents a natural extension of the CLF-based pointwise min-norm concept to a receding horizon methodology, including an appropriate interpretation as a conceptual blend of HJB and Euler-Lagrange philosophies. This interaction of approaches is found to inherit not only the theoretical advantages of each methodology, but unexpectedly results in practical and advantageous implementation properties.

Let $V$ be a CLF and let $u_{\sigma}$ and $x_{\sigma}$ denote the control and state trajectories obtained by solving the pointwise min-norm problem with parameter $\sigma(x)$ [cf. (7), (8)]. Consider the following receding horizon objective:

\section{$(\mathrm{RHC}+\mathrm{CLF})$}

$$
\begin{aligned}
& \inf _{u(\cdot)} \quad \int_{t}^{t+T}\left(q(x)+u^{T} u\right) d \tau \\
& \text { s.t. } \quad \dot{x}=f(x)+g(x) u \\
& \frac{\partial V}{\partial x}[f+g u(t)] \leq-\epsilon \sigma(x(t)) \\
& V(x(t+T)) \leq V\left(x_{\sigma}(t+T)\right)
\end{aligned}
$$

with $0<\epsilon \leq 1$. This optimization is solved on-line and implemented in a receding horizon fashion.

The preceding scheme is best interpreted in the following manner. It is a standard receding horizon formulation with two CLF constraints. The first constraint (16) is a direct stability constraint in the spirit of that which appears in the pointwise min-norm formulation (8). The parameter $\epsilon$ is merely used to relax this constraint as compared to its counterpart in the pointwise min-norm formulation. Note that this constraint need only apply to the implemented control actions, which, if the optimizations are solved "continuously," is only the initial control action. The above RHC+CLF optimization corresponds to this ideal case. On the other hand, since most practical implementations of receding horizon control solve the optimization at discrete sampling instances, the constraint (16) should apply at least over the entire sampling interval in which each optimizing control solution to the RHC+CLF problem is implemented. In essence, this constraint requires $V$ to be a Lyapunov function for the closed-loop system.

In contrast to the first constraint which is a direct stability constraint, the second constraint (17) is oriented toward performance and replaces the terminal weight used in the standard receding horizon formulation. While a terminal weight penalizes

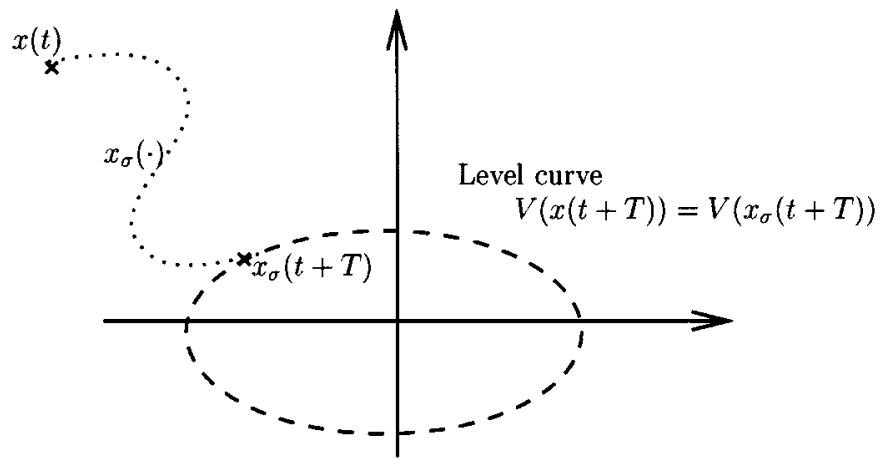

Fig. 2. Performance constraint (17).

large values of the final predicted state, this constraint explicitly restricts the final state. It is obtained by first simulating the controller from the solution to the pointwise min-norm problem for time $T$, which results in a predicted state trajectory that ends at $x_{\sigma}(t+T)$, then evaluating the CLF at this point $\left[V\left(x_{\sigma}(t+T)\right)\right]$. The constraint then requires that all other potential sequences reach a final state that obtains a smaller value of $V$. A nice interpretation is in terms of level curves. The constraint (17) requires that the final state of all potential sequences lie inside the level curve of $V$ that passes through $x_{\sigma}(t+T)$ (see Fig. 2). As will be seen later, when the pointwise min-norm problem corresponding to Sontag's formula is used [i.e., $\sigma=\sigma_{s}$ (9)], this constraint preserves the property that when the level curves of the CLF $(V)$ correspond to those of the value function $\left(V^{*}\right)$, the optimal controller is recovered.

This combination of CLF and receding horizon control yields a number of theoretically appealing properties, as listed below.

1) Stability is guaranteed for any horizon $T$. The constraint (16) requires that $V$ is a Lyapunov function for the receding horizon controlled system and, hence, guarantees stability.

2) In the limit as the horizon goes to zero $(T \rightarrow 0)$, the pointwise min-norm optimization problem is recovered. It was already shown that as $T \rightarrow 0$, the limiting performance objective is given by $u^{T} u$. We only need to show that the constraints reduce to the pointwise min-norm constraint (8). Subtracting $V(x(t))$ from both sides of the performance constraint (17) gives

$V(x(t+T))-V(x(t)) \leq V\left(x_{\sigma}(t+T)\right)-V(x(t))$.

Dividing by $T$ and taking the limit as $T \rightarrow 0$ yields

$$
\begin{aligned}
& \frac{\partial V}{\partial x}[f(x)+g(x) u(t)] \\
& \quad \leq \frac{\partial V}{\partial x}\left[f(x)+g(x) u_{\sigma}(x(t))\right] \\
& \quad \leq-\sigma(x(t)) .
\end{aligned}
$$

In fact, it is simple to see that the constraints

$$
\begin{gathered}
\frac{\partial V}{\partial x}[f(x)+g(x) u(t)] \leq \frac{\partial V}{\partial x}\left[f(x)+g(x) u_{\sigma}(x(t))\right] \\
\text { and } \\
\qquad \frac{\partial V}{\partial x}[f(x)+g(x) u(t)] \leq-\sigma(x(t))
\end{gathered}
$$


produce the same control actions in the pointwise min-norm formulation.

Since we require that $\epsilon \leq 1$ in the stability constraint (16), the above constraint supersedes the stability constraint in the limit. Hence, the receding horizon optimization problem is reduced to

$$
\begin{array}{ll}
\inf _{u} & u^{T}(t) u(t) \\
\text { s.t. } & \frac{\partial V}{\partial x}[f(x)+g(x) u(t)] \leq-\sigma(x) .
\end{array}
$$

3) If $V$ is a Lyapunov function for the closed-loop system under the optimal control, $u^{*}$, and constraint (16) is always satisfied, then an infinite horizon length will always recover the optimal controller. With an infinite horizon $(T=\infty)$, the objective becomes an infinite horizon objective

$$
\int_{t}^{\infty}\left(q(x)+u^{T} u\right) d \tau
$$

With no constraints, the solution to this is the optimal controller $u^{*}$. We only need to show that under the assumptions, the optimal controller is feasible. By assumption, it is feasible for the first constraint (16). For an infinite horizon, the performance constraint (17) becomes that the state must approach zero as $t$ approaches infinity. Clearly, this is satisfied under the optimal controller. Hence, the optimal unconstrained controller is a feasible solution and, therefore, optimal.

The second stability property given above helps to clarify the role of the direct stability constraint (16) and the relaxation parameter $\epsilon$. Note that the stability constraint (16) is identical to the constraint (8) in the pointwise min-norm problem, although with an added parameter $\epsilon$, it applies over the entire range of implemented control actions. The relaxation by $\epsilon$ is allowed for the following reason. From the justification of the second stability property given above, we saw that when the horizon tends to zero, the performance constraint (17) actually reduces to (8), which guarantees stability in the pointwise min-norm formulation. Unfortunately, as the performance constraint (17) in the RHC + CLF scheme, it does not guarantee stability anymore. Hence, we must impose the additional constraint (16) to directly guarantee stability. But, in some sense the information from the parameter $\sigma$ is already contained in (17), so the stability constraint (16) is more of a "backup" and does not need to be as restrictive as (8); hence, the relaxation parameter $\epsilon$.

While we have been somewhat informal about our justification of the above properties, in the Appendix, a rigorous treatment is given. The argument above that the optimization problem reduces to the optimal infinite horizon problem or the pointwise min-norm formulation as the horizon tends to infinity or zero is strengthened to show that the receding horizon control action obtained from the RHC + CLF problem will converge to the optimal control action $u^{*}$ or the pointwise min-norm controller $u_{\sigma}$ as the horizon extends to infinity or shrinks to zero. Details are contained in the Appendix.

Additionally, for the parameter choice $\sigma(x)=\sigma_{s}(x)$ corresponding to Sontag's formula in the pointwise min-norm problem [see (9)], the optimality property of Sontag's formula is preserved.

Theorem IV.1: Let $\sigma(x)=\sigma_{s}(x)$ [cf. (9)]. If $V$ has the same shape level curves as those of the value function $V^{*}$, then the optimal controller is recovered for any horizon length.

Proof: Assume that $V$ has the same shape level curves as the value function $V^{*}$. In this case, Sontag's formula results in an optimal state trajectory $x_{\sigma_{s}}$ and control action $u_{\sigma_{s}}$ [10]. Let us assume that $x_{\sigma_{s}}$ and $u_{\sigma_{s}}$ do not solve the RHC+CLF optimization problem (15)-(17). Hence, feasible trajectories $x(\cdot)$ and $u(\cdot)$ exist such that

$$
\int_{t}^{t+T}\left(q(x)+u^{T} u\right) d \tau<\int_{t}^{t+T}\left(q\left(x_{\sigma_{s}}\right)+u_{\sigma_{s}}^{T} u_{\sigma_{s}}\right) d \tau .
$$

Furthermore, since $x(\cdot)$ and $u(\cdot)$ satisfy (17), we have that

$$
V(x(t+T)) \leq V\left(x_{\sigma_{s}}(t+T)\right)
$$

or using the fact that $V$ has the same shape level curves as $V^{*}$

$$
V^{*}(x(t+T)) \leq V^{*}\left(x_{\sigma_{s}}(t+T)\right) .
$$

Combining (18) and (19) and the fact that Sontag's formula is optimal [10] gives

$$
\begin{aligned}
& \int_{t}^{t+T}\left(q(x)+u^{T} u\right) d \tau+V^{*}(x(t+T)) \\
& \quad< \int_{t}^{t+T}\left(q\left(x_{\sigma_{s}}\right)+u_{\sigma_{s}}^{T} u_{\sigma_{s}}\right) d \tau+V^{*}\left(x_{\sigma_{s}}(t+T)\right) \\
& \quad=V^{*}(x(t))
\end{aligned}
$$

which is a contradiction, since $V^{*}$ is the minimum infinite horizon cost.

Before addressing some of the implementation properties of this new RHC+CLF scheme, let us summarize the key ideas behind this approach. From a practical viewpoint, it involves a mix of the guaranteed stability properties of CLF's combined with the on-line optimization and performance properties of receding horizon control. Conceptually, it blends the philosophies behind the HJB and Euler-Lagrange approaches to the nonlinear optimal control problem. The CLF represents the best approximation to the value function in the HJB approach. The on-line optimization then proceeds in an Euler-Lagrange fashion, optimizing over trajectories emanating from the current state, improving the solution by using as much computation time as is available.

\section{A. Implementation Issues}

In addition to the theoretical properties of the previous section, the RHC + CLF scheme possesses a number of desirable implementation properties.

1) An initial feasible trajectory for the optimization is provided by the solution to the pointwise min-norm problem. For the performance constraint (17), it is necessary to simulate the solution to the pointwise min-norm problem over the horizon $T$ to obtain $x_{\sigma}(t+T)$. Additionally, the control and state trajectory from this pointwise min-norm problem provide an initial feasible trajectory from which to begin the optimization. Note that this pointwise min-norm trajectory is automatically feasible for the 
constraint (16), even when it is applied over a sampling interval, and hence, guarantees that the optimization is always feasible.

2) The optimization may be preempted without loss of stability. Since (16) ensures that $V$ will be a Lyapunov function for the closed-loop system, any control trajectory that satisfies this constraint will be stabilizing. In particular, if the optimization cannot be completed, one may implement the current best solution and proceed without any loss of stability. Hence, there is no requirement of a global optimum to the generally nonconvex optimization (15)-(17) to guarantee stability.

3) The horizon may be varied on-line without loss of stability. This is again due to the stability constraint (16). Since stability is guaranteed by the constraint (16) and is independent of the objective function, it is clear that the horizon may be varied on-line without jeopardizing stability. In particular, one could imagine a situation where the amount of time available for on-line computation is not constant. When more time is available, the horizon can be extended on-line to take advantage of this. On the other hand, if at various times no on-line computation is available, the horizon can be drawn in to zero where the control is given by the pointwise min-norm solution. In essence, one may use the available computation time to its fullest by adjusting the horizon on-line, all without any concern of losing stability.

As mentioned previously, in practice, receding horizon control is typically not implemented in continuous time, but rather at discrete sampling times. Over each sampling interval, the receding horizon control problem is solved and the optimizing control solution is applied until a new state update is received at the next sampling time and the process repeats. Furthermore, (16) applies over the entire sampling interval so that all control actions that are implemented conform to $V$ being a Lyapunov function. There may even be cases in which it is convenient to simply impose the constraint (16) over the entire horizon $[t, t+T]$ (e.g., when sampling intervals are not regularly spaced). Beyond this, when a finite sampling time $T_{s}$ is being used, $\epsilon$ can even be a function of time $\epsilon(\tau), \tau \in[t, t+T]$ satisfying:

1) $\epsilon(\tau) \leq 1$ for all $\tau \in[t, t+T]$

2) $\epsilon(\tau)>0$ for all $\tau \in\left[t, t+T_{s}\right]$.

The amount of relaxation of the stability constraint (16), determined by $\epsilon$, is a design freedom. We typically employ small values of $\epsilon$, since this allows more freedom in the on-line optimizations, placing greater emphasis on on-line computations. Larger values of $\epsilon$ will have the opposite effect, restricting the on-line optimizations to trajectories that are closer to the pointwise min-norm solution over the sampling time.

We should point out that other approaches to guaranteeing stability under sampling that do not require extending the constraint in (16) may exist. In particular, results in [4] and references therein may be relevant to such an approach.

Finally, we mention that receding horizon schemes are often used to address constrained systems. The inclusion of constraints in our framework complicates both the search for a
CLF and the RHC + CLF control scheme as introduced above. While beyond the scope of this paper, readers interested in the issue of constraints are referred to [18], [21], and [9] for advances in the determination of constrained CLF's, and [25] for an extension of the RHC+CLF scheme in this paper to input constrained systems.

In the next section, we demonstrate the RHC + CLF approach on a two-dimensional (2-D) oscillator example.

\section{EXAMPLE}

In this section we present an example that illustrates some of the key properties and limitations of Sontag's formula (10) and receding horizon control, as well as an application of the RHC + CLF scheme. Consider the 2-D nonlinear oscillator

$$
\left\{\begin{aligned}
\dot{x}_{1}= & x_{2} \\
\dot{x}_{2}= & -x_{1}\left(\frac{\pi}{2}+\arctan \left(5 x_{1}\right)\right) \\
& \quad-\frac{5 x_{1}^{2}}{2\left(1+25 x_{1}^{2}\right)}+4 x_{2}+3 u
\end{aligned}\right.
$$

with performance index

$$
\int_{0}^{\infty}\left(x_{2}^{2}+u^{2}\right) d \tau
$$

This example was created using the so-called converse HJB method [5] so that the optimal solution is known. For this problem, the value function is given by

$$
V^{*}=x_{1}^{2}\left(\frac{\pi}{2}+\arctan \left(5 x_{1}\right)\right)+x_{2}^{2}
$$

which results in the optimal feedback law

$$
u^{*}=-3 x_{2} .
$$

A simple technique for obtaining a CLF for this system is to exploit the fact that it is feedback linearizable [12]. In the feedback linearized coordinates, a quadratic function may be chosen as a CLF. In order to ensure that this CLF will at least produce a locally optimal controller, we chose a quadratic CLF that agrees with the quadratic portion of the true value function. ${ }^{1}$ This resulted in the following $\mathrm{CLF}^{2}$

$$
V=\frac{\pi}{2} x_{1}^{2}+x_{2}^{2}
$$

As mentioned earlier, Sontag's formula relies heavily on the shape of the level curves of the CLF. If those shapes are the same as the level curves from the value function, then Sontag's formula is optimal. We have chosen the CLF in this case so that exactly the opposite is true, and the level curves of the CLF deviate far from the level curves of the value function away from the origin. Fig. 3 is a plot of the level curves of the true value function $V^{*}$ versus those of the CLF $V$. The result is that Sontag's formula performs poorly, accumulating a cost of 250 from the

\footnotetext{
${ }^{1}$ This can be done without knowledge of the true value function by performing Jacobian linearization and designing an LQR optimal controller for the linearized system

${ }^{2}$ This function is actually not a CLF in the strict sense in that there exist points where $\dot{V}$ may only be made equal to zero and not strictly less than zero. This is sometimes referred to as a weak CLF. Nevertheless, we will use this CLF since it is the only quadratic function that locally agrees with our value function (which itself is not even a strict CLF for this system). Furthermore, asymptotic stability under Sontag's formula is guaranteed by LaSalle's invariance principle.
} 


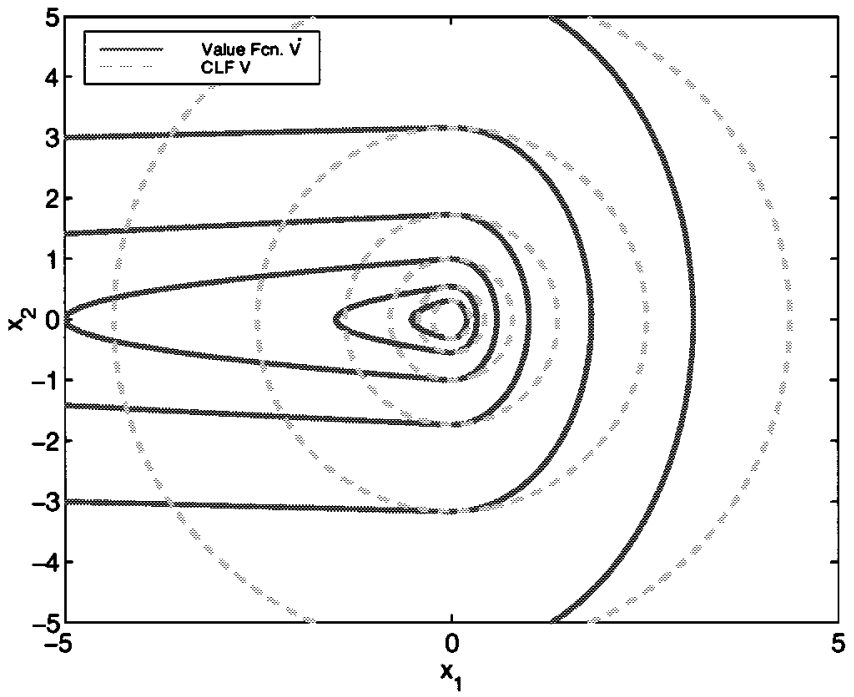

Fig. 3. Contours of the value function (solid) and CLF (dashed).

initial condition $[3,-2]$, in contrast to the optimal controller's cost of 31.7 .

The same initial condition $([3,-2])$ also produces nonintuitive behavior from receding horizon control. Consider a receding horizon scheme with no terminal weight [i.e., $\varphi(x)=0$ ] applied for various values of the horizon $T$. At $T=0.2$, the closed-loop trajectory is unstable from $[3,-2]$. As the horizon is increased to $T=0.3$, the results change dramatically and near optimal performance is achieved by the receding horizon controller. At this point, one might be tempted to assume that a sufficient horizon for stability has been reached and longer horizons would only improve the performance. In actuality the opposite happens, and as the horizon is increased further, the performance deteriorates and returns to instability by a horizon of $T=0.5$. This instability remains present even past a horizon of $T=1.0$.

The RHC+CLF scheme, by combining both the stability properties of Sontag's formula and the performance advantages of receding horizon techniques, avoids the difficulties of the pure CLF and receding horizon controller. Building upon Sontag's formula [i.e., using $\sigma_{s}$ in (9)], a horizon was introduced in accordance with the RHC + CLF scheme (as described in Section IV). In our implementation, the optimizations were resolved at discrete time instances using a sampling time of 0.1 . Furthermore, the stability constraint (16) was applied over this entire 0.1 intersample time using $\epsilon=0.01$. As shown in Fig. 4, the erratic behavior demonstrated by the receding horizon controllers, and the poor performance of Sontag's formula are both absent for all of the tested horizons. Table I summarizes the costs accumulated for each of the horizons $T=0.2,0.3,0.5$, and 1.0 .

The fact that the cost does not decrease monotonically as a function of horizon length is attributable to the erratic behavior that receding horizon control by itself displays. Note that while the RHC+CLF scheme produces excellent costs, they are not guaranteed to be an improvement over a pure receding horizon or CLF-based scheme. In fact, the simple receding horizon controller with no terminal weight and horizon $T=0.3$ performs

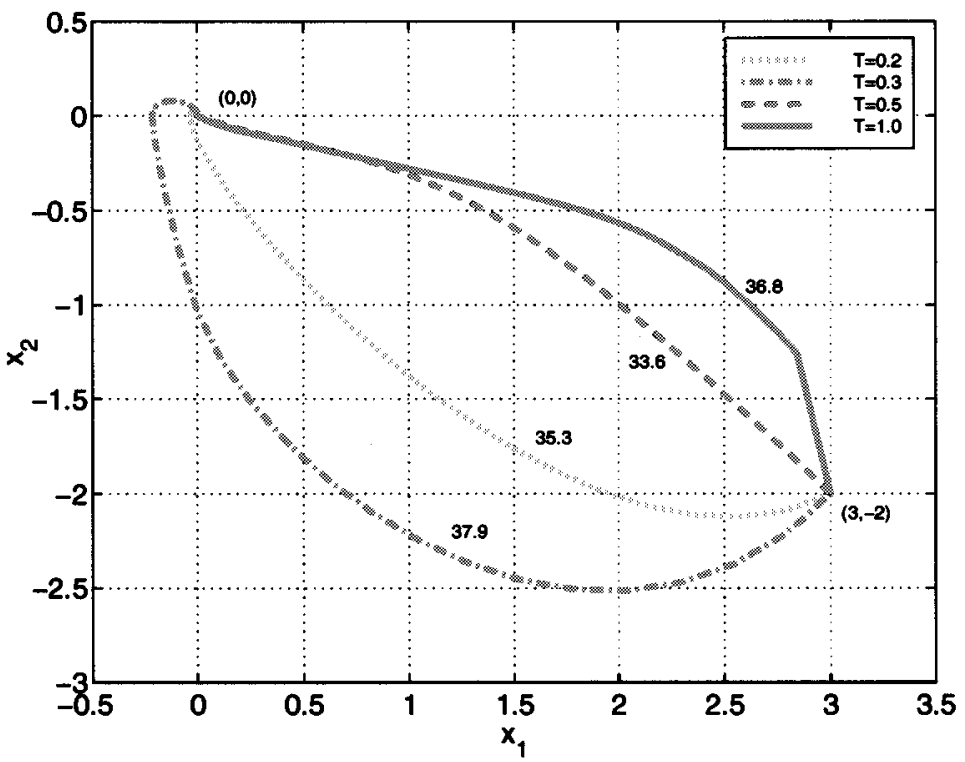

Fig. 4. Phase portrait of RHC+CLF controllers.

TABLE I

SUMmary OF CONTROLLER COSTS FROM INITIAL CONDITION $[3,-2]$

\begin{tabular}{c|c}
\hline Controller & Cost \\
\hline \hline Sontag & 258 \\
RHC+CLF $(T=0.2)$ & 35.3 \\
RHC+CLF $(T=0.3)$ & 37.9 \\
RHC+CLF $(T=0.5)$ & 33.6 \\
RHC+CLF $(T=1.0)$ & 36.8 \\
Optimal & 31.7 \\
\hline \hline
\end{tabular}

as well as the RHC + CLF scheme from the initial condition $[3,-2]$, even though for other horizons it is unstable. Additionally, other formulations of receding horizon control which guarantee stability [13], [16], [19], [20], [22]-[24] as well as other CLF-based schemes, represent viable alternatives. Nevertheless, the RHC+CLF methodology uses both the information in the CLF and receding horizon computation, and intuitively this should provide it with an advantage over each technique individually.

\section{SUMMARY}

The ideas behind CLF-based pointwise min-norm controllers and receding horizon control were combined to create a new class of control schemes. These new results were facilitated by the development of a framework within which both optimal and pointwise min-norm controllers served as limiting cases of receding horizon control. This led us to propose a natural extension of the pointwise min-norm formulation to allow for on-line computation in a receding horizon implementation. In particular, this even provided a receding horizon "extension" of Sontag's formula, and resulted in numerous theoretical and implementation advantages over present CLF and receding horizon methodologies. These were demonstrated on a simple 2-D nonlinear oscillator example. In the end, we hope that these results will help to spawn new directions of research 
that reveal and exploit the synergistic relationships that exist between many of the current approaches to nonlinear control.

\section{APPENDIX}

In this Appendix, we show that the control actions from the RHC+CLF scheme converge to those of the pointwise min-norm controller and the optimal infinite horizon controller as the horizon is brought to zero and infinity, respectively. But first, we begin by establishing some required notation and assumptions.

Let $|\cdot|$ and $|\cdot|_{\infty}$ denote the standard Euclidean and infinity norms on $\mathbb{R}^{N}$. We will assume that both the CLF $V$ and the value function $V^{*}$ are $\mathbf{C}^{1}$ and proper. As before, $x_{\sigma}(\cdot)$ and $u_{\sigma}(\cdot)$ will denote the state and control corresponding to the pointwise min-norm problem, and $x^{*}(\cdot)$ and $u^{*}(\cdot)$ will represent the state and control of the optimal infinite horizon controller. For any optimization with a nonzero horizon, the positive semi-definite cost parameter $q(\cdot)$ will be at least $\mathbf{C}^{0}$, the initial condition will be denoted $x(0)$, and the optimization will be taken over all piecewise $\mathbf{C}^{0}$ functions with the assumption that the infimum is achieved and is unique. The notation $\hat{V}_{T}$ will be used to denote the optimal cost of the RHC + CLF problem with horizon $T$. The corresponding optimizing state and control trajectories will be denoted by $\hat{x}_{T}(\cdot)$ and $\hat{u}_{T}(\cdot)$. As before, the dynamics are

$$
\dot{x}=f(x)+g(x) u=f(x)+\sum_{i=1}^{m} g_{i}(x) u_{i}
$$

with $x \in \mathbb{R}^{n}$ and $u=\left[u_{1}, u_{2}, \cdots, u_{m}\right]^{T} \in \mathbb{R}^{m}$. We will assume that $f: \mathbb{R}^{n} \rightarrow \mathbb{R}^{n}$ is globally Lipschitz with Lipschitz constant $K_{f}$ and each $g_{i}: \mathbb{R}^{n} \rightarrow \mathbb{R}^{n}$ is globally Lipschitz with common Lipschitz constant $K_{g}$.

For the pointwise min-norm problem (7) we will assume the parameter $\sigma(x)$ is continuous, locally Lipschitz, positive definite, and satisfies

$$
x \neq 0, \frac{\partial V}{\partial x} g(x)=0 \Rightarrow \frac{\partial V}{\partial x} f(x)<-\sigma(x) .
$$

Under these conditions, the pointwise min-norm controller $u_{\sigma}(x)$ is also continuous and locally Lipschitz everywhere except possibly at the origin [6]. Hence, for small enough $t$, it satisfies

$$
\left|u_{\sigma}(x(0))-u_{\sigma}(x(t))\right| \leq K t
$$

for some $K$.

To prove connections between the pointwise min-norm problem and the $\mathrm{RHC}+\mathrm{CLF}$ problem, we will require a similar assumption on the control trajectories from the RHC+CLF problems, stated as follows.

A1) Given a fixed initial condition $x(0)$, for all horizons $T$ sufficiently small $\hat{u}_{T}(t)$ is $\mathbf{C}^{0}$ and satisfies the following Lipschitz condition:

$$
\left|\hat{u}_{T}(0)-\hat{u}_{T}(t)\right| \leq K t, \quad \forall t \in[0, T]
$$

for some $K$.

The assumption A1) also provides some regularity on the variation of the state trajectories $\hat{x}_{T}(\cdot)$. To see this, consider the state trajectory $\hat{x}_{T}(\cdot)$ from the $\mathrm{RHC}+\mathrm{CLF}$ problem beginning at state $x(0)$ and assume A1), then for small enough $T$

$$
\begin{aligned}
& \left|\hat{x}_{T}(t)-x(0)\right| \\
& =\left|\int_{0}^{t}\left(f\left(\hat{x}_{T}(s)\right)+g\left(\hat{x}_{T}(s)\right) \hat{u}_{T}(s)\right) d s\right| \\
& \leq \int_{0}^{t}\left(\left|f\left(\hat{x}_{T}(s)\right)\right|+\left|g\left(\hat{x}_{T}(s)\right) \hat{u}_{T}(s)\right|\right) d s \\
& \leq \int_{0}^{t}\left|f\left(\hat{x}_{T}(s)\right)\right| d s \\
& +\int_{0}^{t}\left|\left[g\left(\hat{x}_{T}(s)\right)-g(x(0))+g(x(0))\right] \hat{u}_{T}(s)\right| d s \\
& \leq \int_{0}^{t}\left(\left|f\left(\hat{x}_{T}(s)\right)-f(x(0))\right|+|f(x(0))|\right) d s \\
& +\int_{0}^{t}\left(\sum_{i=1}^{m}\left|\left[g_{i}\left(\hat{x}_{T}(s)\right)-g_{i}(x(0))\right] \hat{u}_{i T}(s)\right|\right) d s \\
& +\int_{0}^{t}\left(\sum_{i=1}^{m}\left|g_{i}(x(0)) \hat{u}_{i T}(s)\right|\right) d s \\
& \leq \int_{0}^{t}\left(\left|f\left(\hat{x}_{T}(s)\right)-f(x(0))\right|+|f(x(0))|\right) d s \\
& +\int_{0}^{t}\left(\sum_{i=1}^{m}\left|g_{i}\left(\hat{x}_{T}(s)\right)-g_{i}(x(0))\right|\left|\hat{u}_{i T}(s)\right|\right) d s \\
& +\int_{0}^{t}\left(\sum_{i=1}^{m}\left|g_{i}(x(0))\right|\left|\hat{u}_{i T}(s)\right|\right) d s \\
& \leq \int_{0}^{t}\left(\left|f\left(\hat{x}_{T}(s)\right)-f(x(0))\right|+|f(x(0))|\right) d s \\
& +\int_{0}^{t}\left(\sum_{i=1}^{m}\left|g_{i}\left(\hat{x}_{T}(s)\right)-g_{i}(x(0))\right|\left(\left|\hat{u}_{T}(0)\right|+K s\right)\right) d s \\
& +\int_{0}^{t}\left(\sum_{i=1}^{m}\left|g_{i}(x(0))\right|\left(\left|\hat{u}_{T}(0)\right|+K s\right)\right) d s \\
& \leq \int_{0}^{t}\left(K_{f}\left|\hat{x}_{T}(s)-x(0)\right|+|f(x(0))|\right) d s \\
& +\int_{0}^{t}\left(\sum_{i=1}^{m} K_{g}\left|\hat{x}_{T}(s)-x(0)\right|\left(\left|\hat{u}_{T}(0)\right|+K s\right)\right) d s \\
& +\int_{0}^{t}\left(\sum_{i=1}^{m}\left|g_{i}(x(0))\right|\left(\left|\hat{u}_{T}(0)\right|+K s\right)\right) d s \\
& \leq \int_{0}^{t}\left(\left(K_{f}+m K_{g}\left(\left|\hat{u}_{T}(0)\right|+K s\right)\right)\left|\hat{x}_{T}(s)-x(0)\right|\right) d s \\
& +\int_{0}^{t}\left(|f(x(0))|+\left(\left|\hat{u}_{T}(0)\right|+K s\right) \sum_{i=1}^{m}\left|g_{i}(x(0))\right|\right) d s \\
& \leq \int_{0}^{t}\left(\left(K_{f}+m K_{g}\left(\left|\hat{u}_{T}(0)\right|+K s\right)\right)\left|\hat{x}_{T}(s)-x(0)\right|\right) d s \\
& +\left(|f(x(0))|+\left|\hat{u}_{T}(0)\right| \sum_{i=1}^{m}\left|g_{i}(x(0))\right|\right) t \\
& +\left(K \sum_{i=1}^{m}\left|g_{i}(x(0))\right|\right) \frac{t^{2}}{2} \text {. }
\end{aligned}
$$


where we have used assumption A1) and that $f$ and $g$ are Lipschitz. If we let

$$
\begin{aligned}
\lambda(t)= & \left(|f(x(0))|+\left|\hat{u}_{T}(0)\right| \sum_{i=1}^{m}\left|g_{i}(x(0))\right|\right) t \\
& +\left(K \sum_{i=1}^{m}\left|g_{i}(x(0))\right|\right) \frac{t^{2}}{2}
\end{aligned}
$$

and

$$
\mu(s)=\left(K_{f}+m K_{g}\left(\left|\hat{u}_{T}(0)\right|+K s\right)\right)
$$

then we have that

$$
\left|\hat{x}_{T}(t)-x(0)\right| \leq \int_{0}^{t} \mu(s)\left|\hat{x}_{T}(s)-x(0)\right| d s+\lambda(t) .
$$

An application of the Gronwall-Bellman Lemma [14] gives

$\left|\hat{x}_{T}(t)-x(0)\right| \leq \lambda(t)+\int_{0}^{t} \lambda(s) \mu(s) \exp \left[\int_{s}^{t} \mu(\tau) d \tau\right] d s$.

This provides an explicit bound for the amount by which $\hat{x}_{T}$ is allowed to vary in time $t$. Finally, we will implicitly assume that all limits, when stated, exist.

A further justification for some of the above assumptions can be made as follows. Optimal control problems are typically solved by representing the control trajectory over a finite dimensional spline space. This involves the choice of a knot sequence [i.e., a nondecreasing sequence $\left(t_{i}\right)$ ] with respect to which the splines are defined. Most splines will allow discontinuities only on the knot sequence and can be chosen to be smooth in between. The optimization is carried out by using the coefficient of each spline basis function as a decision variable. If these coefficients are restricted to lie in some compact set, then Assumption A1) will necessarily be satisfied. These considerations help to make the continuity and Lipschitz assumptions a bit more natural.

The first theorem shows that the control actions obtained from the RHC+CLF problem converge to the pointwise min-norm solution as the horizon is brought to zero.

Theorem A.1: Denote the initial condition for the RHC + CLF optimization problems by $x(0)$, and assume that $\lim _{T \rightarrow 0} \hat{u}_{T}(0)=\hat{u}_{0}$. Under the assumptions stated above, $\hat{u}_{0}=u_{\sigma}(x(0))$ where $u_{\sigma}(x(0))$ solves the corresponding pointwise min-norm problem.

Proof: First we show that $\hat{u}_{0}$ is feasible for the zero horizon problem [i.e., the pointwise min-norm problem with parameter $\sigma(x)$ as in (8)]. For this purpose, it is sufficient to show that

$$
\frac{\partial V}{\partial x}\left[f+g \hat{u}_{0}\right] \leq \frac{\partial V}{\partial x}\left[f+g u_{\sigma}(x(0))\right] .
$$

Since it is known that each $\hat{u}_{T}$ satisfies (17)

$$
V\left(\hat{x}_{T}(T)\right) \leq V\left(x_{\sigma}(T)\right)
$$

subtracting $V(x(0))$ and dividing by $T$ gives

$$
\frac{1}{T}\left[V\left(\hat{x}_{T}(T)\right)-V(x(0))\right] \leq \frac{1}{T}\left[V\left(x_{\sigma}(T)\right)-V(x(0))\right] .
$$

By the definition of a derivative and the chain rule, taking the limit as $T \rightarrow 0$ gives (22). Hence, $\hat{u}_{0}$ is feasible for the zerohorizon (pointwise min-norm) problem.

Now, assume that $\hat{u}_{0} \neq u_{\sigma}(x(0))$. Since $\hat{u}_{0}$ is feasible, we must have that $\hat{u}_{0}^{T} \hat{u}_{0}>u_{\sigma}^{T}(x(0)) u_{\sigma}(x(0))$ (otherwise, this contradicts that $u_{\sigma}(x(0))$ is the unique solution to the zero horizon [pointwise min-norm] problem [6]). This means that for some $\epsilon>0$, we can find a horizon $T^{\prime}$ small enough so that

$$
q(x(0))+u_{\sigma}^{T}(x(0)) u_{\sigma}(x(0))+\epsilon \leq q(x(0))+\hat{u}_{T^{\prime}}^{T}(0) \hat{u}_{T^{\prime}}(0) .
$$

But, by the Lipschitz condition (20) on $\hat{u}_{T}(\cdot)$ and the bound (21) on the rate of variation of the state trajectory $\hat{x}_{T}(\cdot)$ a similar inequality must hold over a small enough horizon $T^{\prime}$. [Note that (21) actually depends on $\hat{u}_{T}(0)$ through $\lambda(t)$ and $\mu(t)$. Furthermore, $\hat{u}_{T}(0)$ is different for each horizon $T$. Nevertheless, we know that $\hat{u}_{T}(0)$ converges to $\hat{u}_{0}$ and, hence, can still guarantee a bound on the rate of variation of $\hat{x}_{T}$ which is independent of the horizon $T$.] Hence, there exists a $T^{\prime}$ sufficiently small so that

$$
q\left(x_{\sigma}(t)\right)+u_{\sigma}^{T}\left(x_{\sigma}(t)\right) u_{\sigma}\left(x_{\sigma}(t)\right)<q\left(\hat{x}_{T^{\prime}}(t)\right)+\hat{u}_{T^{\prime}}^{T}(t) \hat{u}_{T^{\prime}}(t)
$$

for all $t \in\left[0, T^{\prime}\right]$. Integration from zero to $T^{\prime}$ completes the contradiction since $\hat{u}_{T^{\prime}}(t)$ was assumed optimal for this horizon. Hence, $\hat{u}_{0}=u_{\sigma}$.

Before exploring the solution to the RHC + CLF problem as the horizon is increased to $\infty$, we remind the reader of the following definition.

Definition 1: A function $W: \mathbb{R}_{+} \rightarrow \mathbb{R}_{+}$is said to belong to class $\mathcal{K}_{\infty}$ if:

1) it is continuous;

2) $W(0)=0$;

3) it is strictly increasing;

4) $W(s) \rightarrow \infty$ when $s \rightarrow \infty$.

We will require the nonlinear system to satisfy an additional condition. Using notation from [13], we refer to the following as property $C$.

Definition 2: The system $\dot{x}=f(x)+g(x) u$ is said to satisfy property $C$ if there exists a time $T^{c}$, and a $\mathcal{K}_{\infty}$ function $W_{c}$ such that for any $x_{0} \in \mathbb{R}^{n}$, there exist continuous state and control trajectories $\left(x^{c}(t), u^{c}(t)\right)$ such that $x^{c}(0)=x_{0}$ and $x^{c}\left(T^{c}\right)=0$ with

$$
\int_{0}^{T^{c}}\left|\left(x^{c}(t), u^{c}(t)\right)\right| \leq W_{c}\left(\left|x_{0}\right|\right) .
$$

We will say that the system $\dot{x}=f(x)+g(x) u$ locally has property $C$ if property $C$ holds for some neighborhood of the origin. Note that for $q(\cdot)$ locally Lipschitz, local satisfaction of property $C$ implies that

$$
\left.\int_{0}^{T^{c}} q\left(x^{c}(t)\right)+\mid u^{c}(t)\right)\left.\right|^{2} \leq W_{c}^{\prime}\left(\left|x_{0}\right|\right)
$$

is also satisfied locally for some $\mathcal{K}_{\infty}$ function $W_{c}^{\prime}$.

Remark: Property $C$ can be thought of as a weak controllability condition. Consider a linear system: $\dot{x}=A x+B u$ with $(A, B)$ controllable. Then from any initial condition the state can be brought to the origin using the minimum energy control. It can be shown that this will satisfy property $C$ [13]. 
Theorem A.2: Assume that $q(x)$ is continuous, locally Lipschitz and that $q(x) \geq \alpha(|x|)$ where $\alpha$ is $\mathcal{K}_{\infty}$. Additionally, assume that the optimal infinite horizon controller $u^{*}$ satisfies the CLF stability constraint (16). Furthermore, assume that the nonlinear system $\dot{x}=f(x)+g(x) u$ locally satisfies property $C$. Then over any compact set $\mathcal{S}$

$$
\hat{V}_{T}(x) \stackrel{T \rightarrow \infty}{\longrightarrow} V^{*}(x) \text { uniformly. }
$$

Furthermore, if there exists an interval $[0, \beta]$ on which $\hat{u}_{T}(\tau)$ is continuous for each $T$ and $\hat{u}_{T}(\tau) \rightarrow \hat{u}_{\infty}(\tau)$ uniformly, then $\hat{u}_{\infty}(\tau)=u^{*}(\tau)$ for $\tau \in[0, \beta]$.

Proof: To establish notation, recall that $V^{*}$ is the value function corresponding to the optimal cost of the unconstrained infinite horizon optimal control problem with state and control trajectories $x^{*}$ and $u^{*}$. Let $V_{T}^{*}$ denote the cost of applying the infinite horizon optimal control action $u^{*}$, but only over a horizon of length $T$. Finally, recall that $\hat{V}_{T}$ is the optimal cost of the RHC + CLF problem with horizon $T$ and state and control trajectories $\hat{x}_{T}$ and $\hat{u}_{T}$.

Choose $\epsilon>0$ and consider the set $\mathcal{N}=\left\{x: W_{c}^{\prime}(|x|) \leq \epsilon\right\}$ [with $W_{c}^{\prime}(\cdot)$ as in (23)], which contains a neighborhood of the origin. Furthermore, let $\hat{q}>0$ be the infimum of $q(x)$ outside of $\mathcal{N}$. Now, let $\mathcal{S}$ be any compact set and denote the maximum of $V^{*}$ over $\mathcal{S}$ by $v$. Then, for $T>T^{*}=v / \hat{q}$, a $t \in[0, T]$ exists such that the state $x^{*}(t) \in \mathcal{N}$. That is, from any initial condition in $\mathcal{S}$, after $T^{*}$ seconds it is guaranteed that the optimal trajectory $x^{*}(\cdot)$ has intersected $\mathcal{N}$. This is because if there does not exist a $t \in[0, T]$ with $x^{*}(t) \in \mathcal{N}$, then $q\left(x^{*}(t)\right) \geq \hat{q}$ for all $t \in[0, T]$ and, hence

$$
V^{*}(x)>V_{T}^{*}(x)=\int_{0}^{T}\left(q\left(x^{*}(t)\right)+u^{* T}(t) u^{*}(t)\right) d t \geq T \hat{q}>v
$$

which is a contradiction.

Now, for the RHC + CLF problem with horizon $T>T^{*}+T^{c}$, consider the following feasible control actions. Apply $u^{*}$ (this is feasible by assumption) until the state enters $\mathcal{N}$, then use $u^{c}$ (cf., Definition 2) to drive the state to the origin. If $T^{\mathcal{N}} \leq T^{*}$ denotes the first time that $x^{*}(\cdot)$ enters $\mathcal{N}$, then the cost of this trajectory is less than or equal to $V_{T^{\mathcal{N}}}^{*}+W_{c}^{\prime}\left(\left|x^{*}\left(T^{\mathcal{N}}\right)\right|\right)$ which is less than or equal to $V_{T}^{*}+\epsilon$. Furthermore, this trajectory ends at the origin, and hence, also provides an upper bound for the optimal infinite horizon cost, $V^{*}$. From this we can assert the following: for every horizon $T>T^{*}+T^{c}$, we have

$$
V_{T}^{*}+\epsilon \geq V^{*} \geq V_{T}^{*}
$$

and

$$
V_{T}^{*}+\epsilon \geq \hat{V}_{T} \geq V_{T}^{*}
$$

which implies

$$
\left|V^{*}-\hat{V}_{T}\right| \leq \epsilon
$$

proving the first part of the theorem.

The second portion of the theorem follows in three steps:

1) $\hat{x}_{\infty}$ exists and is unique and continuous on $[0, \beta]$ : By assumption, an interval $[0, \beta]$ exists where $\hat{u}_{T}(\tau)$ is continuous and $\hat{u}_{T}(\tau) \rightarrow \hat{u}_{\infty}(\tau)$ uniformly. Hence, $\hat{u}_{\infty}$ is continuous on $[0, \beta]$. Since $[0, \beta]$ is compact, $\hat{u}_{\infty}(t)$ is bounded. Let $\max _{t \in[0, \beta]}\left|\hat{u}_{\infty}(t)\right|_{\infty}=M$.

Now, let $\hat{x}_{\infty}$ be the state trajectory corresponding to the input $\hat{u}_{\infty}$ over the interval $[0, \beta]$. If we define $\hat{f}(x, t)=$ $f(x)+g(x) \hat{u}_{\infty}(t)$ on $t \in[0, \beta]$, then $\hat{f}(x, t)$ is Lipschitz since

$$
\begin{aligned}
& |\hat{f}(x, t)-\hat{f}(y, t)| \\
& \quad=\left|f(x)-f(y)+[g(x)-g(y)] \hat{u}_{\infty}(t)\right| \\
& \quad \leq|f(x)-f(y)|+\left|[g(x)-g(y)] \hat{u}_{\infty}(t)\right| \\
& \quad \leq|f(x)-f(y)|+\sum_{i=1}^{m}\left|\left[g_{i}(x)-g_{i}(y)\right] \hat{u}_{i \infty}(t)\right| \\
& \quad \leq|f(x)-f(y)|+\sum_{i=1}^{m} M\left|\left[g_{i}(x)-g_{i}(y)\right]\right| \\
& \quad \leq|f(x)-f(y)|+\sum_{i=1}^{m} M K_{g}|x-y| \\
& \quad \leq K_{f}|x-y|+m M K_{g}|x-y| \\
& \quad=\left(K_{f}+m M K_{g}\right)|x-y|
\end{aligned}
$$

where we have used that $f$ and $g$ are Lipschitz with Lipschitz constants $K_{f}$ and $K_{g}$, and that $\hat{u}_{\infty}(\cdot)$ is bounded in infinity norm by $M$. Therefore, by standard existence and uniqueness theorems for differential equations (see $[14$, p. 81$])$, the state trajectory $\hat{x}_{\infty}$ exists and is unique and continuous on $[0, \beta]$.

2) $\hat{x}_{T}$ converges to $\hat{x}_{\infty}$ on $[0, \beta]$ : Let us show that $\hat{x}_{T}$ converges pointwise to $\hat{x}_{\infty}$ on $[0, \beta]$. This is basically an exercise in using Lipschitz constants, and an application of the Gronwall-Bellman lemma ([14, p. 68]).

$$
\begin{aligned}
& \left|\hat{x}_{\infty}(t)-\hat{x}_{T}(t)\right| \\
& =\mid \int_{0}^{t}\left(f\left(\hat{x}_{\infty}(s)\right)-f\left(\hat{x}_{T}(s)\right)\right) d s \\
& +\int_{0}^{t}\left(g\left(\hat{x}_{\infty}(s)\right) \hat{u}_{\infty}(s)-g\left(\hat{x}_{T}(s)\right) \hat{u}_{T}(s)\right) d s \mid \\
& \leq \int_{0}^{t}\left(\left|f\left(\hat{x}_{\infty}(s)\right)-f\left(\hat{x}_{T}(s)\right)\right|\right) d s \\
& +\int_{0}^{t}\left(\left|g\left(\hat{x}_{\infty}(s)\right) \hat{u}_{\infty}(s)-g\left(\hat{x}_{T}(s)\right) \hat{u}_{T}(s)\right|\right) d s \\
& =\int_{0}^{t}\left(\left|f\left(\hat{x}_{\infty}(s)\right)-f\left(\hat{x}_{T}(s)\right)\right|\right) d s \\
& +\int_{0}^{t}\left(\left|g\left(\hat{x}_{\infty}(s)\right) \hat{u}_{\infty}(s)-g\left(\hat{x}_{\infty}(s)\right) \hat{u}_{T}(s)\right|\right) d s \\
& +\int_{0}^{t}\left(\left|g\left(\hat{x}_{\infty}(s)\right) \hat{u}_{T}(s)-g\left(\hat{x}_{T}(s)\right) \hat{u}_{T}(s)\right|\right) d s \\
& \leq \int_{0}^{t}\left(K_{f}\left|\hat{x}_{\infty}(s)-\hat{x}_{T}(s)\right|\right) d s \\
& +\int_{0}^{t}\left(\sum_{i=1}^{m}\left|g_{i}\left(\hat{x}_{\infty}(s)\right)\left[\hat{u}_{i \infty}(s)-\hat{u}_{i T}(s)\right]\right|\right) d s \\
& +\int_{0}^{t}\left(\sum_{i=1}^{m}\left|\left[g_{i}\left(\hat{x}_{\infty}(s)\right)-g_{i}\left(\hat{x}_{T}(s)\right)\right] \hat{u}_{i T}(s)\right|\right) d s
\end{aligned}
$$




$$
\begin{aligned}
\leq & \int_{0}^{t}\left(K_{f}\left|\hat{x}_{\infty}(s)-\hat{x}_{T}(s)\right|\right) d s \\
& +\int_{0}^{t}\left(\sum_{i=1}^{m}\left|g_{i}\left(\hat{x}_{\infty}(s)\right)\right|\left|\left[\hat{u}_{i \infty}(s)-\hat{u}_{i T}(s)\right]\right|\right) d s \\
& +\int_{0}^{t}\left(\sum_{i=1}^{m}\left|\left[g_{i}\left(\hat{x}_{\infty}(s)\right)-g_{i}\left(\hat{x}_{T}(s)\right)\right]\right|\left|\hat{u}_{i T}(s)\right|\right) d s .
\end{aligned}
$$

Now, note that each $\left|g_{i}\left(\hat{x}_{\infty}(s)\right)\right|$ is bounded from above on $[0, \beta]$ since it is a continuous function over a compact set. Hence, choose an $M_{g}$ such that $\max _{t \in[0, \beta]}\left|g_{i}\left(\hat{x}_{\infty}(t)\right)\right| \leq M_{g}$ for $i=1 \cdots m$. Furthermore, by the fact that $\hat{u}_{T}$ converges uniformly to $\hat{u}_{\infty}$, by choosing $T$ large enough we can bound $\max _{t \in[0, \beta]}\left|\hat{u}_{T}(\cdot)\right|_{\infty}$ by $M+1$ [recall that $\left.\max _{t \in[0, \beta]}\left|\hat{u}_{\infty}(\cdot)\right|_{\infty}=M\right]$. Hence, returning to our bound

$$
\begin{aligned}
\left|\hat{x}_{\infty}(t)-\hat{x}_{T}(t)\right| & \\
\leq & \int_{0}^{t}\left(K_{f}\left|\hat{x}_{\infty}(s)-\hat{x}_{T}(s)\right|\right) d s \\
& +\int_{0}^{t}\left(\sum_{i=1}^{m}\left|g_{i}\left(\hat{x}_{\infty}(s)\right)\right|\left|\left[\hat{u}_{i \infty}(s)-\hat{u}_{i T}(s)\right]\right|\right) d s \\
& +\int_{0}^{t}\left(\sum_{i=1}^{m}\left|\left[g_{i}\left(\hat{x}_{\infty}(s)\right)-g_{i}\left(\hat{x}_{T}(s)\right)\right]\right| \hat{u}_{i T}(s) \mid\right) d s \\
\leq & \int_{0}^{t}\left(K_{f}\left|\hat{x}_{\infty}(s)-\hat{x}_{T}(s)\right|\right) d s \\
& +\int_{0}^{t}\left(\sum_{i=1}^{m} M_{g}\left|\hat{u}_{i \infty}(s)-\hat{u}_{i T}(s)\right|\right) d s \\
& +\int_{0}^{t}\left(\sum_{i=1}^{m}(M+1) K_{g}\left|\hat{x}_{\infty}(s)-\hat{x}_{T}(s)\right|\right) d s \\
\leq & \int_{0}^{t}\left(\left(K_{f}+m(M+1) K_{g}\right)\left|\hat{x}_{\infty}(s)-\hat{x}_{T}(s)\right|\right) d s \\
& +\int_{0}^{t}\left(m M_{g}\left|\hat{u}_{\infty}(s)-\hat{u}_{T}(s)\right|_{\infty}\right) d s .
\end{aligned}
$$

Now, let $\epsilon=\max _{t \in[0, \beta]}\left|\hat{u}_{\infty}(t)-\hat{u}_{T}(t)\right|_{\infty}$. Since $\hat{u}_{T}$ converges uniformly to $\hat{u}_{\infty}$, then $\epsilon \rightarrow 0$ as $T \rightarrow \infty$. So

$$
\begin{aligned}
& \left|\hat{x}_{\infty}(t)-\hat{x}_{T}(t)\right| \\
& \quad \leq \int_{0}^{t}\left(\left(K_{f}+m(M+1) K_{g}\right)\left|\hat{x}_{\infty}(s)-\hat{x}_{T}(s)\right|\right) d s+m M_{g} \epsilon t .
\end{aligned}
$$

By an application of the Gronwall-Bellman lemma, we obtain

$$
\begin{aligned}
\left|\hat{x}_{\infty}(t)-\hat{x}_{T}(t)\right| & \\
\leq & m M_{g} \epsilon t \\
+ & \int_{0}^{t}\left(m M_{g} \epsilon s\left(K_{f}+m(M+1) K_{g}\right)\right. \\
\cdot & \left.e^{\left(K_{f}+m(M+1) K_{g}\right)(t-s)}\right) d s \\
=\epsilon & {\left[m M_{g} t+\int_{0}^{t}\left(m M_{g} s\left(K_{f}+m(M+1) K_{g}\right)\right.\right.} \\
& \left.\left.\cdot e^{\left(K_{f}+m(M+1) K_{g}\right)(t-s)}\right) d s\right]
\end{aligned}
$$

which tends to zero as $\epsilon$ approaches zero. Hence, $\hat{x}_{T}$ converges pointwise to $\hat{x}_{\infty}$ on $[0, \beta]$ as $T \rightarrow \infty$ (in fact, the convergence is uniform).

3) $\left(\hat{x}_{\infty}, \hat{u}_{\infty}\right)$ satisfies the principle of optimality. By definition, the cost $\hat{V}_{T}(x(0))$ can be written in terms of $\hat{x}_{T}$ and $\hat{u}_{T}$ as

$$
\hat{V}_{T}(x(0))=\int_{0}^{T}\left(q\left(\hat{x}_{T}(t)\right)+\hat{u}_{T}^{T}(t) \hat{u}_{T}(t)\right) d t
$$

where $\hat{x}_{T}$ and $\hat{u}_{T}$ satisfy (16) and (17). By the principle of optimality, $\hat{x}_{T}(\tau)$ and $\hat{u}_{T}(\tau)$ for $\tau \in[\beta, T]$ solves the optimization problem

minimize

$u[\beta, T]$

subject to

$$
\int_{\beta}^{T}\left(q(x)+u^{T} u\right) d \tau
$$

$$
\begin{aligned}
& \dot{x}=f(x)+g(x) u \\
& x(\beta)=\hat{x}_{T}(\beta) \\
& V(x(T)) \leq V\left(x_{\sigma}(T)\right) .
\end{aligned}
$$

[The only difference between this problem and the RHC + CLF problem is that the stability constraint (16) is absent since it applies only to the initial control action at time zero (i.e., $\hat{u}_{T}(0)$ ).] Let us denote the optimal cost of this problem by $\tilde{V}_{T-\beta}\left(\hat{x}_{T}(\beta)\right)$. By an argument identical to that given for $\hat{V}_{T}$, we can also prove that $\tilde{V}_{T}$ converges uniformly to $V^{*}$ on any compact set. Furthermore, a restatement of the principle of optimality is that

$$
\begin{aligned}
& \hat{V}_{T}(x(0)) \\
& \quad=\int_{0}^{\beta}\left(q\left(\hat{x}_{T}(t)\right)+\hat{u}_{T}^{T}(t) \hat{u}_{T}(t)\right) d t+\tilde{V}_{T-\beta}\left(\hat{x}_{T}(\beta)\right) .
\end{aligned}
$$

Now, take the limit as $T \rightarrow \infty$. On the left-hand side of (24), from the first part of this theorem, we have that

$$
\hat{V}_{T}(x(0)) \rightarrow V^{*}(x(0)) .
$$

Now, consider the right-hand side of (24). We can show that the second term on the right-hand side converges to $V^{*}\left(\hat{x}_{\infty}(\beta)\right)$ as follows:

$$
\begin{aligned}
& \left|V^{*}\left(\hat{x}_{\infty}(\beta)\right)-\tilde{V}_{T-\beta}\left(\hat{x}_{T}(\beta)\right)\right| \\
& \quad \leq\left|V^{*}\left(\hat{x}_{\infty}(\beta)\right)-V^{*}\left(\hat{x}_{T}(\beta)\right)\right| \\
& \quad+\left|V^{*}\left(\hat{x}_{T}(\beta)\right)-\tilde{V}_{T-\beta}\left(\hat{x}_{T}(\beta)\right)\right| .
\end{aligned}
$$

The term

$$
\left|V^{*}\left(\hat{x}_{\infty}(\beta)\right)-V^{*}\left(\hat{x}_{T}(\beta)\right)\right|
$$

tends to zero since $V^{*}$ is continuous and $\hat{x}_{T}(\beta)$ converges to $\hat{x}_{\infty}(\beta)$. Additionally, the term

$$
\left|V^{*}\left(\hat{x}_{T}(\beta)\right)-\tilde{V}_{T-\beta}\left(\hat{x}_{T}(\beta)\right)\right|
$$

tends to zero since by choosing $T$ large enough, we can assert that $\hat{x}_{T}(\beta)$ lies in a compact set [this is because $\hat{x}_{T}(\beta)$ is a convergent sequence]. As mentioned earlier, by the same argument as for $\hat{V}_{T}$ in the first portion of this theorem, we can assert that $\tilde{V}_{T-\beta}$ converges uniformly to 
$V^{*}$ on any compact set. Therefore, this term also tends to zero. So, we conclude that

$$
\tilde{V}_{T-\beta}\left(\hat{x}_{T}(\beta)\right) \rightarrow V^{*}\left(\hat{x}_{\infty}(\beta)\right) .
$$

Finally, we consider the limit of the first term on the right-hand side of (24)

$$
\lim _{T \rightarrow \infty} \int_{0}^{\beta}\left(q\left(\hat{x}_{T}(t)\right)+\hat{u}_{T}^{T}(t) \hat{u}_{T}(t)\right) d t .
$$

The dominated convergence theorem [28] justifies an exchange of the limit and integral. By assumption $\hat{u}_{T} \rightarrow$ $\hat{u}_{\infty}$ and by step $2 . \hat{x}_{T} \rightarrow \hat{x}_{\infty}$. Hence, this term converges to

$$
\int_{0}^{\beta}\left(q\left(\hat{x}_{\infty}(t)\right)+\hat{u}_{\infty}^{T}(t) \hat{u}_{\infty}(t)\right) d t
$$

Therefore, taking the limit as $T \rightarrow \infty$ of (24) gives

$$
V^{*}(x(0))=\int_{0}^{\beta}\left(q\left(\hat{x}_{\infty}(t)\right)+\hat{u}_{\infty}^{T}(t) \hat{u}_{\infty}(t)\right) d t+V^{*}\left(\hat{x}_{\infty}(\beta)\right)
$$

which shows by the principle of optimality that $\hat{u}_{\infty}$ is optimal for the infinite horizon problem over the interval $[0, \beta]$.

\section{REFERENCES}

[1] Z. Artstein, "Stabilization with relaxed controls," Nonlinear Anal., vol. 7, no. 11, pp. 1163-1173, 1983.

[2] A. E. Bryson and Y. Ho, Applied Optimal Control. Washington, DC: Hemisphere, 1975.

[3] H. Chen and F. Allgöwer, "A quasiinfinite horizon nonlinear model predictive control scheme for constrained nonlinear systems," in Proc. 16th Chinese Control Conf., Qindao, China, 1996, pp. 309-316.

[4] F. Clarke, Y. Ledyaev, E. Sontag, and A. Subbotin, "Asymptotic controllability implies feedback stabilization," Automatica, vol. 42, no. 10, pp. 1394-1407, Oct. 1997.

[5] J. C. Doyle, J. Primbs, B. Shapiro, and V. Nevistić, "Nonlinear games: Examples and counterexamples," in Proc. 35th IEEE Conf. Decision and Control, Kobe, Japan, Dec. 1996, pp. 3915-3920.

[6] R. Freeman and P. Kokotović, "Optimal nonlinear controllers for feedback linearizable systems," in Proc. 1995 American Control Conf., Seattle, WA, June 1995, pp. 2722-2726.

[7] —-, "Inverse optimality in robust stabilization," SIAM J. Contr. Optim., vol. 34, pp. 1365-1391, July 1996.

[8] — Robust Nonlinear Control Design. Boston, MA: Birkhäuser, 1996.

[9] R. Freeman and L. Praly, "Integrator backstepping for bounded controls and control rates," IEEE Trans. Automat. Contr., vol. 43, pp. 258-262, Feb. 1998.

[10] R. Freeman and J. Primbs, "Control lyapunov functions: New ideas from an old source," Proc. 35th IEEE Conf. Decision and Control, pp. 3926-3931, Dec. 1996.

[11] C. E. García, D. M. Prett, and M. Morari, "Model predictive control: Theory and practice-A survey," Automatica, vol. 25, pp. 335-348, May 1989.

[12] A. Isidori, Nonlinear Control Systems, 3rd ed, New York: SpringerVerlag, 1995.

[13] S. Keerthi and E. Gilbert, "Optimal infinite-horizon feedback laws for a general class of constrained discrete-time systems: Stability and moving-horizon approximations," J. Optim. Theory Appl., pp. 265-293, 1988.

[14] H. K. Khalil, Nonlinear Systems. New York: Macmillan, 1992.

[15] M. Krstić, I. Kanellakopoulos, and P. Kokotović, Nonlinear and Adaptive Control Design. New York: Wiley, 1995.

[16] W. H. Kwon, A. N. Bruckstein, and T. Kailath, "Stabilizing state feedback design via the moving horizon method," Int. J. Contr., vol. 37, no. 3, pp. 631-643, 1983
[17] W. H. Kwon and A. E. Pearson, "A modified quadratic cost problem and feedback stabilization of a linear system," IEEE Trans. Automat. Contr., vol. AC-22, no. 5, pp. 838-842, 1977.

[18] W. Lin, "Input saturation and global stabilization by output feedback for affine systems," in Proc. 33rd IEEE Conf. Decision and Control, Lake Buena Vista, FL, Dec. 1994, pp. 1323-1328.

[19] D. Q. Mayne, "Nonlinear model predictive control," in Proc. Int. Symposium on the Assessment and Future Research Directions of Nonlinear Model Predictive Control, Ascona, Switzerland, 1998, pp. 77-90.

[20] D. Q. Mayne and H. Michalska, "Receding horizon control of nonlinear systems," IEEE Trans. Automat. Contr., vol. 35, pp. 814-824, July 1990.

[21] F. Mazenc and L. Praly, "Adding integrations, saturated controls, and stabilization of feedforward systems," IEEE Trans. Automat. Contr., vol. 41, pp. 1559-1578, Nov. 1996.

[22] E. S. Meadows, M. A. Henson, J. W. Eaton, and J. R. Rawlings, "Receding horizon control and discontinuous state feedback stabilization," Int. J. Contr., vol. 62, no. 5, pp. 1217-1229, 1995.

[23] H. Michalska and D. Q. Mayne, "Robust receding horizon control of constrained nonlinear systems," IEEE Trans. Automat. Contr., vol. 38, pp. 1623-1633, Nov. 1993

[24] G. De Nicolao, L. Magni, and R. Scattolini, "Stabilizing nonlinear receding horizon control via a nonquadratic terminal penalty," in IMACS Multiconference CESA'96, Lille, France, July 1996.

[25] J. A. Primbs and M. Giannelli, "A control Lyapunov function based receding horizon methodology for input constrained nonlinear systems," in Int. Federation of Automatic Control, 14th World Congr., Beijing, China, July 1999.

[26] J. Richalet, "Industrial applications of model based predictive control," Automatica, vol. 29, pp. 1251-1274, 1993

[27] J. Richalet, A. Rault, J. L. Testud, and J. Papon, "Model predictive heuristic control: Applications to industrial processes," Automatica, vol. 14, no. 5, pp. 413-428, 1978.

[28] H. L. Royden, Real Analysis. New York: Macmillan, 1988.

[29] R. Sepulchre, M. Jankovic, and P. Kokotovic, Constructive Non-linear Control. New York: Springer-Verlag, 1997.

[30] E. D. Sontag, "A Lyapunov-like characterization of asymptotic controllability," SIAM J. Contr. Optim., vol. 21, no. 3, pp. 462-471, 1983.

[31] — "A "universal" construction of Artstein's theorem on nonlinear stabilization," Syst. Contr. Lett., vol. 13, no. 2, pp. 117-123, 1989.

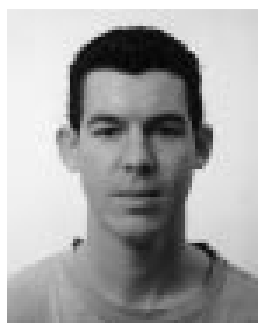

James A. Primbs received B.S. degrees in electrical engineering and mathematics from the University of California, Davis, in 1994, the M.S. degree in electrical engineering from Stanford University, CA, in 1995, and the Ph.D. degree in control and dynamical systems from the California Institute of Technology (Caltech), Pasadena, in 1999.

$\mathrm{He}$ is currently a post-doctoral Researcher in the Control and Dynamical Systems Department at Caltech. His research interests include nonlinear control, complex systems, and finance.

Vesna Nevistić received the Dipl. degree from the Department of Electronics at the Military Technical Academy in Zagreb in 1988, the M.S. degree from the Electrical Engineering Department at Zagreb University in 1991, and the Ph.D. in electrical engineering from the Swiss Federal Institute of Technology (ETH), Zurich, Switzerland, in 1997.

She currently works as an Engagement Manager for McKinsey and Co., Inc., Zurich, Switzerland, primarily focusing on corporate finance and strategy topics.

Dr. Nevistić received the Asea Brown Boweri award for her dissertation research.

John C. Doyle (M'96) received the B.S. and M.S. degrees in electrical engineering from the Massachusetts Institute of Technology, Cambridge, and the $\mathrm{Ph} . \mathrm{D}$. in mathematics from the University of California, Berkeley.

$\mathrm{He}$ is currently the Professor in control and dynamical systems at the California Institute of Technology, Pasadena. His main research interest is in robustness of complex systems. He has received several prize paper awards, including 2 Axelby and a Baker prize from the IEEE. 